\title{
Disrupted salience network functional connectivity and white-matter microstructure in persons at risk for psychosis: findings from the LYRIKS study
}

\author{
C. Wang ${ }^{1}$, F. Ji ${ }^{1}$, Z. Hong ${ }^{1}$, J. S. Poh ${ }^{1}$, R. Krishnan ${ }^{1}$, J. Lee ${ }^{2,3}$, G. Rekhi ${ }^{2}$, R. S. E. Keefe ${ }^{4}$, \\ R. A. Adcock ${ }^{4,5}$, S. J. Wood ${ }^{6,7}$, A. Fornito ${ }^{8}$, O. Pasternak ${ }^{9}$, M. W. L. Chee ${ }^{1}$ and J. Zhou ${ }^{1,10 *}$ \\ ${ }^{1}$ Center for Cognitive Neuroscience, Neuroscience and Behavioral Disorder Program, Duke-NUS Medical School, National University of Singapore, \\ Singapore \\ ${ }^{2}$ Research Division, Institute of Mental Health, Singapore \\ ${ }^{3}$ Office of Clinical Sciences, Duke-NUS Medical School, Singapore \\ ${ }^{4}$ Department of Psychiatry and Behavioral Sciences, Duke University, Durham, NC, USA \\ ${ }^{5}$ Center for Cognitive Neuroscience, Duke University, Durham, NC, USA \\ ${ }^{6}$ School of Psychology, University of Birmingham, Edgbaston, UK \\ ${ }^{7}$ Department of Psychiatry, Melbourne Neuropsychiatry Centre, University of Melbourne and Melbourne Health, Victoria, Australia \\ ${ }^{8}$ Monash Clinical and Imaging Neuroscience, School of Psychology and Psychiatry E Monash Biomedical Imaging, Monash University, Australia \\ ${ }^{9}$ Departments of Psychiatry and Radiology, Brigham and Women's Hospital, Harvard Medical School, Boston, MA, USA \\ ${ }^{10}$ Clinical Imaging Research Centre, the Agency for Science, Technology and Research and National University of Singapore, Singapore
}

Background. Salience network (SN) dysconnectivity has been hypothesized to contribute to schizophrenia. Nevertheless, little is known about the functional and structural dysconnectivity of SN in subjects at risk for psychosis. We hypothesized that SN functional and structural connectivity would be disrupted in subjects with At-Risk Mental State (ARMS) and would be associated with symptom severity and disease progression.

Method. We examined 87 ARMS and 37 healthy participants using both resting-state functional magnetic resonance imaging and diffusion tensor imaging. Group differences in SN functional and structural connectivity were examined using a seed-based approach and tract-based spatial statistics. Subject-level functional connectivity measures and diffusion indices of disrupted regions were correlated with CAARMS scores and compared between ARMS with and without transition to psychosis.

Results. ARMS subjects exhibited reduced functional connectivity between the left ventral anterior insula and other SN regions. Reduced fractional anisotropy (FA) and axial diffusivity were also found along white-matter tracts in close proximity to regions of disrupted functional connectivity, including frontal-striatal-thalamic circuits and the cingulum. FA measures extracted from these disrupted white-matter regions correlated with individual symptom severity in the ARMS group. Furthermore, functional connectivity between the bilateral insula and FA at the forceps minor were further reduced in subjects who transitioned to psychosis after 2 years.

Conclusions. Our findings support the insular dysconnectivity of the proximal SN hypothesis in the early stages of psychosis. Further developed, the combined structural and functional SN assays may inform the prognosis of persons at-risk for psychosis.

Received 3 January 2016; Revised 12 May 2016; Accepted 16 May 2016; First published online 11 July 2016

Key words: At risk for psychosis, functional connectivity, LYRIKS, salience network, transition to psychosis, white-matter microstructure.

\section{Introduction}

Schizophrenia is increasingly viewed as a disease that involves the dysconnectivity of brain networks

* Address for correspondence: Dr J. Zhou, Center for Cognitive Neuroscience, Neuroscience \& Behavioral Disorders Program, Duke-NUS Medical School, 8 College Road, \#06-15, Singapore 169857, Singapore.

(Email: helen.zhou@duke-nus.edu.sg)
(Pettersson-Yeo et al. 2011; Fornito et al. 2012). Functional network architecture characterized by intrinsic connectivity networks (ICNs) has been widely studied in patients with schizophrenia. ICNs are defined as coherent patterns of low-frequency fluctuations in blood oxygen level-dependent signals derived from task-free functional magnetic resonance imaging (fMRI; Zhou et al. 2010). The salience network (SN) is one of the major ICNs and consists of the fronto-insula, anterior cingulate cortex (ACC), orbital frontal cortex

This is an Open Access article, distributed under the terms of the Creative Commons Attribution licence (http://creative commons.org/licenses/by/4.0/), which permits unrestricted re-use, distribution, and reproduction in any medium, provided the original work is properly cited. 
(OFC), striatum, as well as limbic structures (Seeley et al. 2007). The functional integration of nodes within the $\mathrm{SN}$ is crucial to sustain human emotion and cognition, especially during the detection and processing of salient information (Seeley et al. 2007; Craig, 2009; Menon \& Uddin, 2010). Dysfunction of the SN has been hypothesized to occur in schizophrenia, resulting in inappropriate neural responses to internal and external stimuli. These inappropriate neural responses are thought to eventually lead to positive psychotic symptoms such as hallucination and delusions (Williamson, 2007; Palaniyappan \& Liddle, 2012; Palaniyappan et al. 2013; Zhou \& Seeley, 2014).

SN-related abnormalities in schizophrenia have been consistently found in neuroimaging studies. A number of imaging studies have reported reductions in graymatter volume (Saze et al. 2007; Fornito et al. 2009; Takahashi et al. 2009) and task-related activation (Minzenberg et al. 2009; Wilmsmeier et al. 2010) and connectivity (Schmidt et al. 2016) within the insula and ACC in schizophrenia. Task-free fMRI studies have demonstrated reduced functional connectivity (FC) within the insula (Liang et al. 2006; Zhou et al. 2007), ACC (Boksman et al. 2005; Honey et al. 2005) and other key SN regions. Diffusion tensor imaging (DTI) has been used to examine altered white-matter (WM) microstructures in schizophrenia. Reduction in DTI indices purported to quantify WM diffusion properties, such as fractional anisotropy (FA) and axial diffusivity (AD) in frontal and temporal regions (forming part of the $\mathrm{SN}$ ) has been reported in schizophrenia (Buchsbaum et al. 2006) using voxel-based morphometry (Le Bihan, 2003) and region-of-interest (ROI; Price et al. 2005; Li et al. 2014) approaches as well as tract-based spatial statistics (TBSS; Lee et al. 2012; Liu et al. 2013). Furthermore, diffusion tractography studies have directly demonstrated altered structural connectivity within key SN regions in schizophrenia patients (Oh et al. 2009; Bracht et al. 2014).

Recently, there has been growing interest in the study of adolescents and young adults who are in the putative prodromal stage of schizophrenia and are experiencing subthreshold psychotic symptoms. These individuals are at high risk of transitioning to clinical psychosis over a 36-month period and are labeled as having an 'At-Risk Mental State' (ARMS; Yung et al. 1996; Fusar-Poli et al. 2012). Several functional and structural imaging studies have attempted to examine changes in FC and diffusion in WM within ARMS subjects (Wood et al. 2013); these studies have reported mixed results. Both increases and decreases in FC were found across multiple ICNs in ARMS subjects. In one seed-based task-free fMRI study, ARMS subjects showed increased FC within the default mode network (DMN; Shim et al. 2010), while another recent study found reduced FC associated with the dorsal striatum and preserved FC in the ventral striatum (Dandash et al. 2014). DTI studies of ARMS subjects have found reduced FA in frontal regions, the anterior limb of the internal capsule, the posterior cingulate and the angular gyrus (Hoptman et al. 2008; Karlsgodt et al. 2009; Peters et al. 2009; von Hohenberg et al. 2014). Increased FA has also been reported in the ACC and the right middle and superior frontal gyri (Hoptman et al. 2008) as well as in the superior longitudinal fasciculus (Schmidt et al. 2015). Although these inconsistent findings may reflect true heterogeneity in ARMS patients, they may also be attributed to the small sample sizes used in the aforementioned studies. Moreover, the majority of previous studies have examined ARMS patient samples in North America, Europe and Australia. There is a dearth of DTI and functional connectivity studies in East Asian populations. As culture plays an important role in defining and shaping the presentation of psychotic symptoms in patients (Laroi et al. 2014), here we aim to fill this gap by studying a group of Asian ARMS participants in Singapore. Another advantage of conducting this research in Singapore is the relatively low prevalence of drug use (United Nations Office on Drugs and Crime, 2008). Substance use is a problematic confound in ARMS research in Western countries (Habets et al. 2011; Welch et al. 2011) and has been shown to have the potential to affect both structural and functional brain integrity (Liao et al. 2012; Edward Roberts et al. 2014). Our recent work found no gray-matter volume reduction in substance-free ARMS subjects; this finding contrasts with results found for Western ARMS subjects with a high prevalence of drug use (Klauser et al. 2015). Therefore, the study of ARMS subjects who have minimal exposure to substance use allows for a more precise characterization of symptom-related brain connectivity changes.

In addition to examining the structural and functional integrity of the SN in ARMS subjects, another aim of the present study is to investigate whether any of the SN abnormalities identified in ARMS subjects could be used to predict individual clinical outcomes. Several neuroimaging studies have found baseline differences between ARMS individuals who transition to psychosis (ARMS-T) and those who do not (ARMS-NT) in cortical thickness (Tognin et al. 2013), WM integrity (Bloemen et al. 2010; Carletti et al. 2012) and task-related activations (Allen et al. 2012). These studies highlighted structural and functional changes in the prefrontal cortex, anterior insula (AI), temporal lobe and subcortical structures as potential features associated with transition. However, little is known about whether and how ICNs predict the transition to psychosis. 
To this end, we tested the functional and structural SN dysconnectivity hypothesis in a relatively large East Asian sample of 96 drug-free ARMS subjects and 46 healthy controls (HCs) using both task-free fMRI and DTI approaches. We predicted that the FC and integrity of WM microstructure of $\mathrm{SN}$ would be reduced in ARMS individuals and that the degree of alteration would correlate with symptom severity. Within the ARMS group, we also expected that compared to ARMS-NT, the ARMS subgroup who transitioned to psychosis would have more reductions of FC and WM microstructure disruptions in the SN.

\section{Method}

\section{Participants}

We studied 96 ARMS subjects and 46 HCs (aged 14-29 years). The participants were assigned to the ARMS group if they met specific criteria based on the results of assessment with the Comprehensive Assessment of At-Risk Mental States (CAARMS; Yung et al. 2005; Yaakub et al. 2013). The study protocol for this investigation was approved by the National Healthcare Group Domain-Specific Review Board. Further details are included in the Supplementary Methods.

Participants were excluded from the study if they had a history of neurological or serious medical disorder, had a diagnosis of mental retardation, or were taking any antipsychotic medications. Participants with a current history of illicit substance use were also excluded from the study. Fifty-two of ARMS participants were taking prescription antidepressants. Age-matched HCs were recruited from the community if they had no history of neuropsychiatric disorder and no family history of psychosis in first-degree relatives.

\section{Image acquisition}

The participants underwent one neuroimaging session on a 3-T Siemens Tim Trio system (Siemens, Germany). The session included the acquisition of high-resolution T1-weighted images $(\mathrm{TR} / \mathrm{TE}=2300 / 3 \mathrm{~ms}$, voxel size $=$ $1.0 \times 1.0 \times 1.0 \mathrm{~mm}^{3}$ ) during a 6-min task-free fMRI scan $(\mathrm{TR} / \mathrm{TE}=2000 / 30 \mathrm{~ms}$, voxel size $=3.0 \times 3.0 \times 3.0$ $\mathrm{mm}^{3}$; participants were instructed to close their eyes and not fall asleep) and two runs of diffusion MRI $(\mathrm{TR} / \mathrm{TE}=9600 / 107 \mathrm{~ms}$, voxel size $=2.0 \times 2.0 \times 2.0$ $\mathrm{mm}^{3}, 30$ non-collinear diffusion directions with $b=$ $1000 \mathrm{~s} / \mathrm{mm}^{2}, 6$ volumes at $b=0 \mathrm{~s} / \mathrm{mm}^{2}$ ).

\section{FC analyses}

The task-free fMRI data were preprocessed using procedures outlined in a previous study (Zuo et al. 2012) using the FMRIB Software Library (FSL; Jenkinson et al. 2012) and the Analysis of Functional NeuroImages software (Cox, 1996) (see Supplementary Methods). A total of 87 of the 96 ARMS participants and 37 of the $46 \mathrm{HCs}$ passed both the T1 and fMRI quality control measures.

$\mathrm{AI}$ is the key region in the $\mathrm{SN}$ underlying salience processing and introceptive awareness (Seeley et al. 2007; Uddin, 2015). We examined SN FC integrity using the seed-based FC approach based on AI. To account for structural and functional differences in AI subregions (Mesulam \& Mufson, 1982; Mutschler et al. 2009; Wager \& Barrett, 2004) and the lateralization of dysconnectivity reported in psychosis (Wang et al. 2004; Kunimatsu et al. 2008), we estimated whole-brain voxel-wise FC using four AI seeds that covered the ventral and dorsal fields of the AI bilaterally; these seeds were chosen based on an independent metaanalysis of task-based fMRI studies of insular function (Kurth et al. 2010) (Supplementary Table S1, Supplementary Fig. S1). Group differences in FC to each of the four AI seeds were examined using twosample $t$ tests with a height threshold of $p<0.05$ and cluster threshold of $p<0.05$ family-wise error (FWE) corrected.

\section{Motion scrubbing}

To further investigate if any observed group differences in FC were caused by head motion, we applied motion scrubbing on task-free fMRI data by computing frame displacement (FD) and variance of temporal derivative of time-courses over voxels (DVARS) from each subject's task-free fMRI data, following the steps outlined in a previous study (Power et al. 2012). We discarded fMRI volumes with $\mathrm{FD}>0.2 \mathrm{~mm}$, or DVARS $>0.3 \%$ (Power et al. 2013) and repeated the FC analysis with motion-scrubbed data.

\section{WM microstructural analyses}

The diffusion MRI data were preprocessed and analyzed using the TBSS pipeline (Smith et al. 2006) following our previous approach (Cortese et al. 2013; Hong et al. 2015) (see Supplementary Methods). A total of 81 ARMS participants and 36 HCs passed the quality check for DTI data. A recent study indicated that early-stage schizophrenia was characterized by excessive extracellular-free water, which could result in a biased estimation of DTI metrics (Pasternak et al. 2012). We thus implemented additional steps to derive DTI metrics after accounting for partial volume effects contributed by extracellular free water. This was achieved using a modified bi-tensor model to estimate the diffusion properties of WM tissue and surrounding free water separately (Pasternak et al. 2009). Group differences in the skeletonized images of the free-water 
corrected DTI metrics (FA, AD, mean diffusivity, radial diffusivity) were examined using non-parametric permutation tests at a threshold of $p<0.05$ (threshold-free cluster enhancement corrected) (Smith et al. 2006). From the thresholded $t$ statistic maps, the anatomical locations of significant WM clusters were identified using the JHU WM tractography atlas (Hua et al. 2008).

\section{Correlations with clinical severity}

Based on the identified regions of disrupted SN functional connectivity in ARMS subjects, we assessed the relationships between the mean FC measures in those brain ROIs and symptom severity across ARMS subjects (see Supplementary Methods). Similarly, to evaluate the relationship between altered WM microstructure and clinical symptom severity, we extracted the subject-level mean DTI metrics from the WM clusters that showed group differences and examined their association with CAARMS scores in ARMS.

\section{Comparisons of FC and WM microstructure in ARMS subgroups}

All ARMS subjects were followed clinically for 24 months after the neuroimaging study. During the follow-up period, 10 out of 79 ARMS subjects transitioned to psychosis (eight males; mean age $=21.5 \pm 3.5$ years). Psychosis transition was defined according to the criteria described in CAARMS and required the presence of frank psychotic symptoms for at least 1 week (Yung et al. 2005). Based on the identified ROIs showing group difference between ARMS and controls, we next compared the ROI-based FC and DTI metrics between ARMS-T and ARMS-NT subgroups using two-sample $t$ tests. Age, gender, handedness and ethnicity were included as covariates in all statistical analyses.

\section{Results}

\section{Participant characteristics}

There were no differences in age, handedness, gender and motion parameters (both absolute displacement and frame displacement) between the ARMS and HC groups $(p<0.05)$ (Table 1 , Supplementary Table S2). There was a higher proportion of ethnic Chinese individuals in the ARMS group compared to the HC group $(p=0.003)$. Furthermore, we found no differences in demographics nor motion parameters between ARMS-NT and ARMS-T groups, except a higher proportion of ethnic Indian individuals in the ARMS-NT group compared to the ARMS-T group $(p=0.045)$.

\section{Disrupted AI FC in ARMS subjects}

ARMS participants had reduced FC to the left ventral anterior insula (vAI) compared to HC subjects (Fig. 1a, Supplementary Table S3), involving several $\mathrm{SN}$ regions (left ACC, right posterior insula, bilateral OFC, bilateral putamen/caudate nucleus, right brainstem) and the right middle temporal gyrus (MTG). We found no regions of reduced FC to the other three seeds in ARMS subjects. These observations remain highly similar after motion scrubbing (Supplementary Fig. S2). No regions showing increased FC in the ARMS group with or without motion scrubbing.

\section{Disrupted WM microstructure in ARMS}

We found disrupted WM microstructural measures in ARMS subjects compared to controls. Specifically, ARMS subjects had reduced FA in the left cingulum, left side of the corpus callosum, left uncinate fasciculus (UF), forceps minor, left inferior fronto-occipital fasciculus (IFOF), left superior longitudinal fasciculus, and left anterior thalamic radiation (ATR) (Fig. 1b, Supplementary Table S4). Similarly, reduced AD of ARMS was primarily found along the anterior to posterior axis of the bilateral cingulum/corpus callosum (Fig. 1c, Supplementary Table S4). WM regions that showed microstructural abnormalities in ARMS were close to those gray-matter regions that displayed reduced FC to the left vAI (Fig. 2).

Conversely, no increase in FA or AD was found in ARMS subjects compared to HCs. Additionally, we found no differences in mean diffusivity and radial diffusivity between ARMS and HC subjects.

\section{Correlation between WM microstructure and symptom severity}

Symptom severity, as measured by CAARMS total severity score, was correlated with decreased FA within the left IFOF $(r=-0.355, p<0.05$ FWE corrected), the left UF $(r=-0.311, p<0.05$ FWE corrected) and the left ATR $(r=-0.297, p<0.05$ FWE corrected $)$ in ARMS subjects (Fig. 3). We found no association between CAARMS total severity scores and the FC of $\mathrm{SN}$ regions that showed disrupted $\mathrm{SN}$ connectivity in ARMS.

\section{Difference in functional and structural disruptions between ARMS-T and ARMS-NT}

Among the brain regions that showed reduced FC in ARMS subjects compared to HCs, we found that FC between the left vAI and the right insula was further reduced in ARMS-T subjects compared to ARMS-NT subjects $(t=-3.413, p<0.05$ FWE corrected) (Fig. 4, 
Table 1. Characteristics of individuals with At-Risk Mental State (ARMS) and healthy control participants in fMRI functional connectivity analysis

\begin{tabular}{|c|c|c|c|c|c|c|}
\hline & \multicolumn{2}{|c|}{$\begin{array}{l}\text { ARMS } \\
(N=87)\end{array}$} & \multicolumn{2}{|c|}{$\begin{array}{l}\text { Healthy controls } \\
(N=37)\end{array}$} & \multicolumn{2}{|c|}{ Test statistics } \\
\hline & $N$ & $\%$ & $N$ & $\%$ & $x^{2}$ & $p$ \\
\hline Male & 58 & 66.7 & 20 & 54.1 & 1.77 & 0.18 \\
\hline \multicolumn{7}{|l|}{ Handedness } \\
\hline Right & 76 & 87.4 & 32 & 86.5 & 0.99 & 0.61 \\
\hline Left & 5 & 5.7 & 1 & 2.7 & & \\
\hline Mixed & 6 & 6.9 & 4 & 10.8 & & \\
\hline Ethnicity $^{*}$ & & & & & 14.97 & 0.00 \\
\hline Chinese & 62 & 71.3 & 16 & 43.2 & & \\
\hline Malay & 19 & 21.8 & 9 & 24.3 & & \\
\hline Indian & 5 & 5.7 & 10 & 27.0 & & \\
\hline Other & 1 & 1.1 & 2 & 5.4 & & \\
\hline \multirow[t]{2}{*}{ Medication } & 52 & 59.8 & 0 & 0.0 & - & - \\
\hline & Mean & S.D. & Mean & S.D. & $t$ tests & $p$ \\
\hline Age (years) & 21.5 & 3.6 & 22.3 & 4.0 & -0.92 & 0.36 \\
\hline \multicolumn{7}{|c|}{ Positive and Negative Syndrome Scale } \\
\hline Positive subscale score & 10.8 & 2.9 & & & & \\
\hline Negative subscale score & 11.9 & 4.0 & & & & \\
\hline General psychopathology score & 25.5 & 7.3 & & & & \\
\hline Total score & 48.2 & 12.0 & & & & \\
\hline \multicolumn{7}{|l|}{ CAARMS* } \\
\hline Total severity & 7.3 & 3.4 & 0.2 & 0.4 & 19.38 & 0.00 \\
\hline Total frequency & 8.3 & 4.5 & 0.2 & 0.7 & 16.30 & 0.00 \\
\hline \multicolumn{7}{|l|}{ Head motion parameters } \\
\hline Absolute displacement (max) & 0.59 & 0.56 & 0.74 & 0.71 & -1.25 & 0.21 \\
\hline Absolute displacement (mean) & 0.19 & 0.14 & 0.24 & 0.16 & -1.77 & 0.08 \\
\hline Frame displacement (max) & 0.30 & 0.39 & 0.42 & 0.59 & -1.10 & 0.28 \\
\hline Frame displacement (mean) & 0.05 & 0.03 & 0.06 & 0.03 & -0.61 & 0.54 \\
\hline
\end{tabular}

CAARMS, Comprehensive Assessment of At-Risk Mental States.

$t$ tests and $\chi^{2}$ tests were used to assess group differences in continuous and discrete variables, respectively. ${ }^{*}$ Represents a significant difference in ethnicity composition and CAARMS scores between ARMS subjects and healthy controls $(p<0.05)$. The demographic information of subjects included in DTI analysis is presented in Supplementary Table S2 (81 ARMS and 29 controls overlapped with the fMRI cohort).

left). Similarly, among the WM regions that showed ARMS-associated FA reductions, the FA of the forceps minor was more reduced in ARMS-T subjects compared to ARMS-NT subjects $(t=-2.977, p<0.05$ FWE corrected) (Fig. 4, right). In contrast, we found no group difference in CAARMS total severity between ARMS-T and ARMS-NT ( $p<0.05$ corrected).

\section{Co-morbid depression, anxiety disorder and antidepressant medication}

To investigate the FC and WM microstructural differences that could be related to anxio-depressive disorders and antidepressant usage, we further compared
FC and WM FA values in disrupted regions between ARMS subjects who had a concomitant diagnosis of depression and/or anxiety $(N=52)$ and ARMS subjects who did not $(N=35)$. We found no significant differences between the two groups. A similar comparison between ARMS individuals who were taking antidepressants and those who were not also did not reveal any significant differences.

\section{Discussion}

Although there is evidence for the SN dysconnectivity hypothesis in schizophrenia, whether the hypothesis 


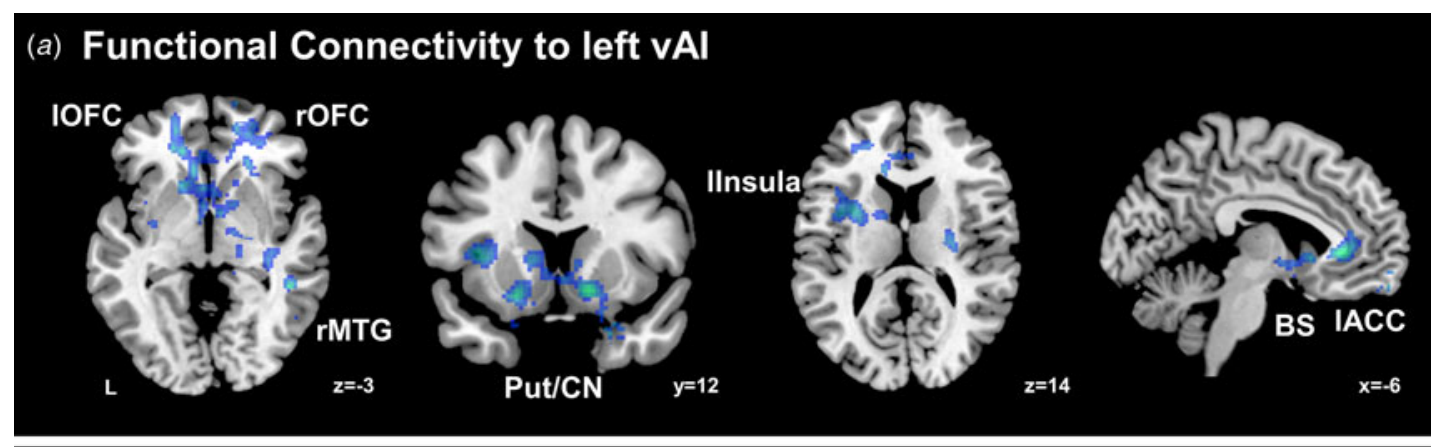

\section{(b) Fractional Anisotropy}

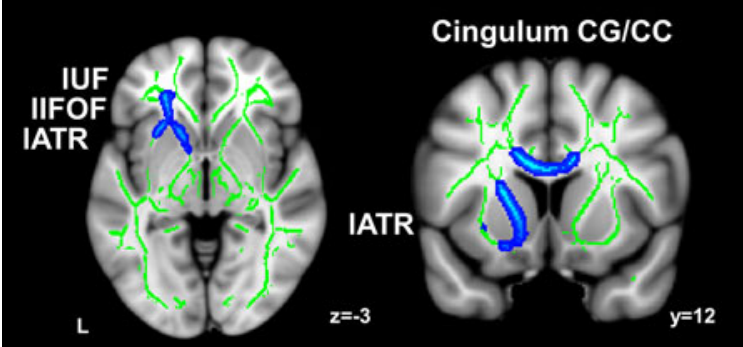

(c) Axial Diffusivity

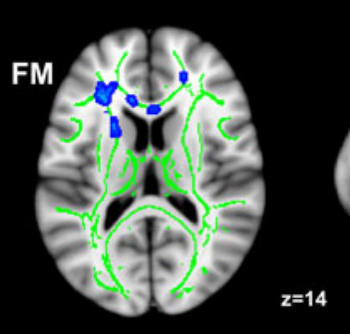

ICingulum CG/CC

Fig. 1. Reduced functional connectivity (FC) to the left ventral anterior insula and disrupted white-matter integrity in At-Risk Mental State (ARMS) subjects compared to healthy controls. Top row: (a) Group-level differences (ARMS < controls) in FC using seeds in the left ventral anterior insula were reported (at a height threshold of $p<0.05$ and cluster threshold of $p<0.05$ FWE corrected). No FC increase was found in ARMS compared to controls. Bottom row: white-matter tracts where ARMS subjects showed reduced fractional anisotropy $(b)$ and axial diffusivity $(c)$ compared to controls were reported (regions highlighted in blue) at $p<0.05$ threshold-free cluster enhancement corrected. ACC, Anterior cingulate cortex; ATR, anterior thalamic radiation; BS, brainstem; CC, corpus callosum; CG, cingulate gyrus; CN, caudate nucleus; FM, forceps minor; IFOF, inferior fronto-occipital fasciculus; MTG, middle temporal gyrus; OFC, Orbital frontal cortex; Put, putamen; UF, uncinate fasciculus; l, left; r, right.

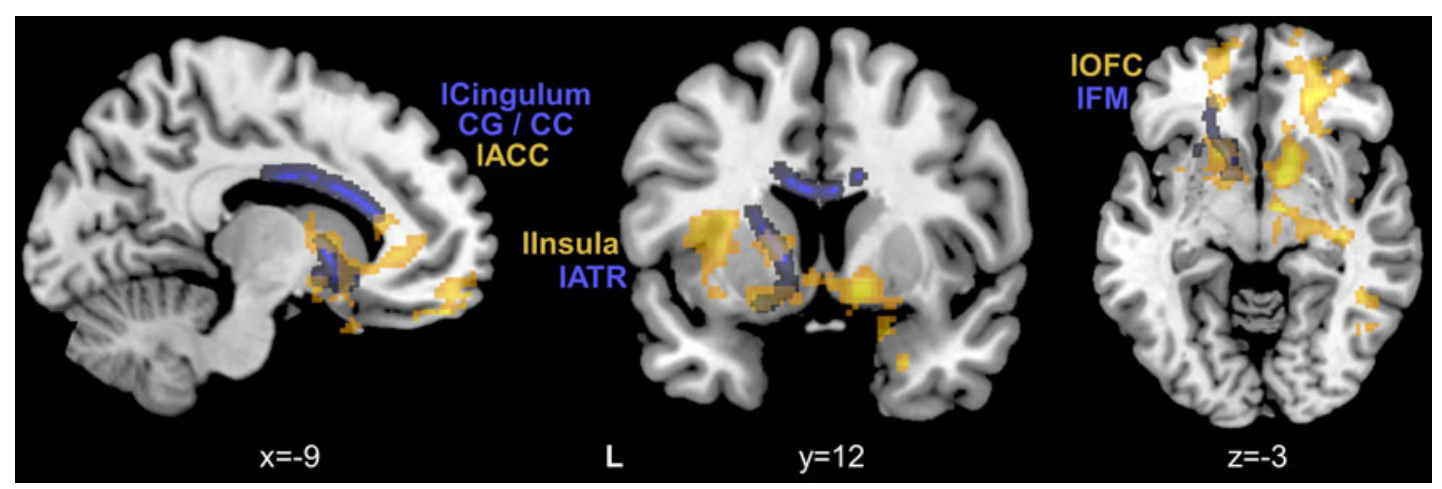

Fig. 2. Structural dysconnectivity in At-Risk Mental State (ARMS) is closely linked to salience network functional connectivity reductions. Compared to healthy controls, ARMS participants had reduced fractional anisotropy in white-matter tracts (highlighted in blue), which were close to those brain regions (highlighted in orange) showing reduced salience network functional connectivity to the left ventral anterior insula in ARMS. ACC, anterior cingulate cortex; ATR, anterior thalamic radiation; CC, corpus callosum; CG, cingulate gyrus; FM, forceps minor; OFC, orbital frontal cortex; l, left; r, right.

extends to at-risk subjects remains unclear. We used both task-free fMRI and DTI to test the SN dysconnectivity hypothesis in a large sample of 96 substance-free ARMS subjects. Relative to age-matched HCs, ARMS subjects showed reduced FC between the left vAI and several SN regions. The ARMS group also showed reduced FA and $\mathrm{AD}$ in fronto-striatal-thalamic circuits close to the regions that showed reduced FC. Furthermore, the disruptions in WM microstructure related to the severity of attenuated psychotic symptoms in ARMS subjects. These SN disruptions and the related WM microstructure alternations also predicted transition to psychosis. Taken together, our findings support the hypothesis of 

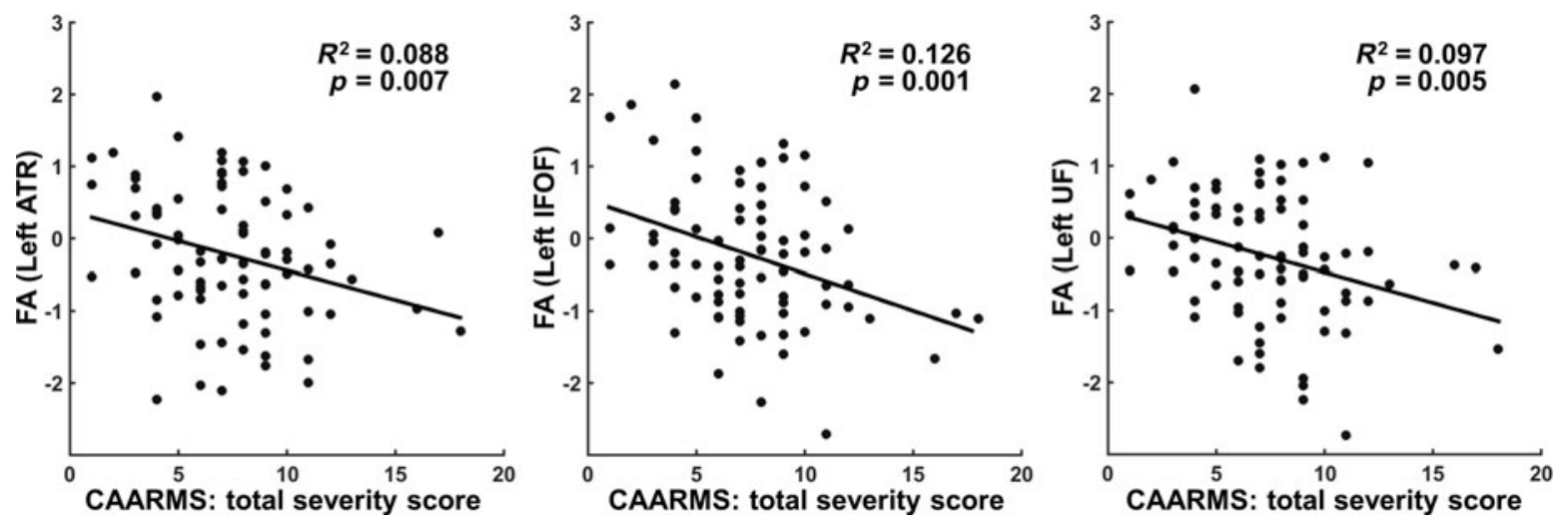

Fig. 3. White-matter integrity disruption correlates with symptom severity. Clinical severity evaluated by Comprehensive Assessment of At-Risk Mental States (CAARMS) was negatively correlated with fractional anisotropy (FA) values in the white-matter regions with group difference (Fig. 2) $(p<0.05$ FWE corrected). FA values in scatter plots are standardized residuals after controlling for age, gender, handedness and ethnicity. ATR, Anterior thalamic radiation; IFOF, inferior fronto-occipital fasciculus; UF, uncinated fasciculus.
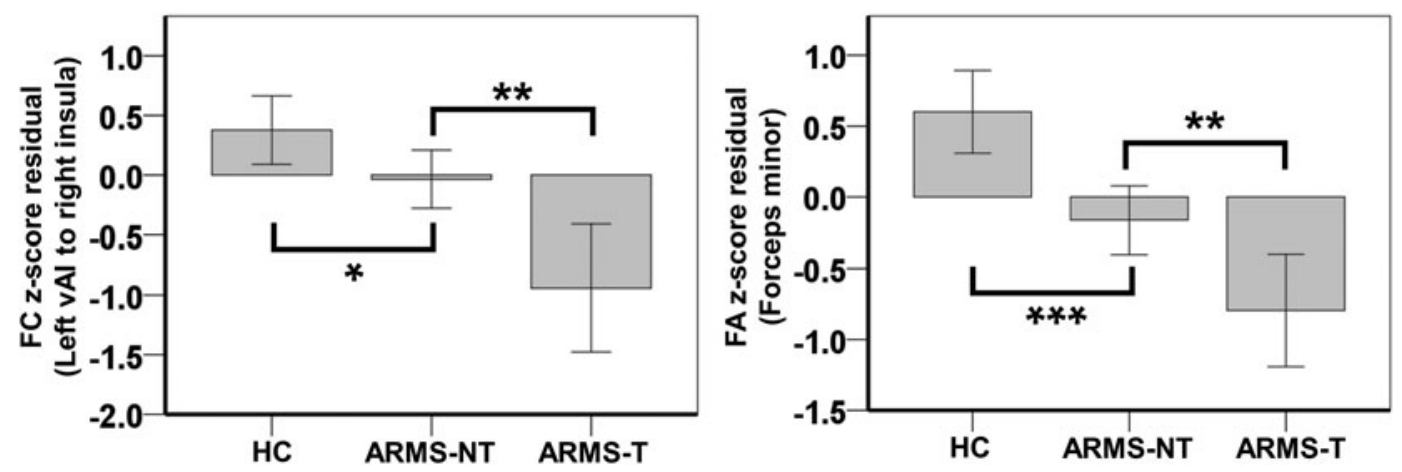

Fig. 4. Functional and structural dysconnectivity predicted psychotic conversion in At-Risk Mental State (ARMS) subjects. Bar charts showing FC between left vAI and right insula as well as FA in the forceps minor of healthy controls (HC), ARMS subjects who transitioned to psychosis (ARMS-T) and ARMS subject who did not make the transition (ARMS-NT). FC or FA values were standardized residuals after controlling for age, gender, handedness and ethnicity. Error bars represent $95 \%$ confidence intervals. The significance of pairwise group differences is indicated by ${ }^{*} p<0.05,{ }^{* *} p<0.01$ and ${ }^{* *} p<0.001$. The comparisons between HC and ARMS-T are significant at $p<0.001$ for both plots (not marked). All passed the multiple comparison correction $(p<0.05)$, except the contrast between HC and ARMS-NT for FC. FA, Fractional anisotropy; FC, functional connectivity; vAI, ventral anterior insula.

network-level disruptions of the salience system in ARMS participants.

\section{Reduced $v A I$ FC in ARMS}

In the ARMS group, key $\mathrm{SN}$ regions, including the right insula, left ACC, bilateral OFC, bilateral striatum, and brainstem, were found to exhibit reduced intrinsic FC with the vAI. These findings are in agreement with previous task-free fMRI studies reporting SN intrinsic connectivity disruptions in schizophrenia (Fusar-Poli, 2012; Tu et al. 2012; Mamah et al. 2013; Wood et al. 2013). Because the AI performs a wide range of neurocognitive functions and is functionally differentiated (Wager \& Barrett, 2004; Mutschler et al. 2009), it is not surprising that AI subfields did not contribute equally to SN dysconnectivity in ARMS. Our findings highlighted the left vAI as the focal point of network disruptions in pre-clinical psychosis. The vAI has been shown to be involved in the regulation of emotionally related physiological states and to serve as an intermediary for functional associations between other insular regions and the limbic system (Krolak-Salmon et al. 2003; Mutschler et al. 2009). This could explain our finding that most of the FC disruptions involving the left vAI also involved regions that are closely associated with the limbic system, including the ACC, bilateral insula, and striatum regions.

Emerging evidence demonstrated dysconnectivity between $\mathrm{SN}$ and other ICNs in patients with 
schizophrenia (Jafri et al. 2008; Mamah et al. 2013; Palaniyappan et al. 2013; Wotruba et al. 2014) and reduced long-range FC in early onset schizophrenia (Yang et al. 2014; Jiang et al. 2015; Li et al. 2015). We found reduced FC between left vAI and right MTG, usually considered to be part of the DMN (Greicius et al. 2004; Fox et al. 2005), in the ARMS group. The impaired interactions between specific regions of $\mathrm{SN}$ and DMN may be reduced in ARMS participants, which suggest that persons at-risk for psychosis might have difficulty coordinating between externally guided and internally monitored states, leading to subthreshold psychotic symptoms (Menon \& Uddin, 2010; Zhou \& Seeley, 2014).

\section{Disrupted WM microstructure in fronto-striatal-thalamic circuits correlated with symptom severity in ARMS}

Reduced FA along major WM tracts connecting the thalamus, basal ganglia and prefrontal cortex was observed in ARMS subjects, indicating the disruption of WM microstructure in fronto-striatal-thalamic circuits. Importantly, we found an association between WM microstructure (i.e. FA measures in the left ATR, left IFOF and left UF) and psychotic symptom severity, as measured by the CAARMS. This type of WM microstructure disruption has been reported from first-episode psychosis (Federspiel et al. 2006; PerezIglesias et al. 2010) to chronic illness (Liu et al. 2013, 2014; Oh et al. 2009); however, few studies have been conducted among ARMS subjects (Wood et al. 2013). Fronto-striatal-thalamic circuits include important neural pathways, such as the ATR and fronto-striatal circuits. The latter circuits are involved in executive and other higher cognitive domains (Van der Werf et al. 2003; Chudasama \& Robbins, 2006). Disruptions of the ATR have been associated with cognitive abnormalities and negative symptoms in patients with schizophrenia (Mamah et al. 2010). One task-related PET imaging study suggested a link between dysfunction in fronto-striatal systems and prodromal signs of psychosis, especially related to executive functions (Fusar-Poli et al. 2010). Early fronto-striatal circuit dysfunction such as that observed here could thus contribute to cognitive impairment and reduced social functioning (Fornito et al. 2013; Dandash et al. 2014). Notably, we observed a clear trend towards greater WM disruption in the left hemisphere in ARMS. Indeed, a few studies have reported reduced FA leftward asymmetry along the anterior cingulum and UF in schizophrenia patients (Wang et al. 2004; Kubicki et al. 2007; Kunimatsu et al. 2008). The current findings suggest a left hemisphere-dominated WM disruption pattern in early stages of psychosis. Whether and how such pattern contributes to changes in WM cerebral asymmetry requires further validation (Oertel-Knochel \& Linden, 2011).

Reduced AD was primarily observed in localized regions of the cingulum, corpus callosum and ATR. Disruptions in the cingulum were previously associated with inefficient cognitive functioning in schizophrenia (Perez-Iglesias et al. 2010; Tang et al. 2010). The cingulum is critical for efficient information processing, as it links three key brain networks (Kelly et al. 2008; Leech et al. 2011). The cingulum connects frontal regions (including $\mathrm{SN}$ ) and posterior DMN regions (i.e. the posterior cingulate cortex), and the hippocampal portion of the cingulum projects from the posterior cingulate cortex to the limbic system (i.e. hippocampus).

The concordance between disrupted FC in the SN and WM microstructure in ARMS subjects strengthens the case for aberrant SN connectivity. Using TBSS analysis, we identified altered diffusion properties along major WM tracts, which provide critical structural support to SN function. Specifically, reduced FA and AD were evident along the left ATR, forceps minor and corpus callosum. These WM tracts project into SN regions showing reduced FC in prefrontal cortex, cingulate gyrus and striatum. Although the TBSS analysis did not directly measure the strength of structural connectivity between these regions, it evaluated the diffusion properties specifically along major WM tracts in the brain, closely approximating the integrity of structural foundation supporting FC between distant brain regions (Smith et al. 2006). Thus using two different neuroimaging modalities, our findings showed converging evidences supporting the SN dysconnectivity hypothesis in ARMS subjects.

\section{FC and WM microstructure in ARMS subjects predicted clinical progression}

Based on these converging functional and structural alternations in a high-risk group, an important question is whether we could use these assays to predict individual's transition to psychosis. Here, we found specific ARMS-associated changes (i.e. FC between bilateral insula and FA of forceps minor changes at baseline) that associated with subsequent psychotic conversion. Notably, the forceps minor is a WM tract that connects the lateral and medial part of the frontal lobes bilaterally; thus could be the structural mechanism that explain the reduced functional synchrony between SN regions in the left and right hemisphere. To date, a number of studies have provided evidence that dysfunction in task-related networks (Sabb et al. 2010; Allen et al. 2012) as well as whole-brain voxel-wise WM changes (Bloemen et al. 2010; Carletti et al. 2012) may foreshadow the onset of psychosis, but few have 
examined the changes in ICNs or network-based WM microstructures. Our findings extended these studies by demonstrating the involvement of brain networks in task-free conditions and corresponding changes in WM tracts. Since task-free fMRI could be more readily implemented in clinical settings than task-related studies, our work demonstrates that specific neuroimaging features could potentially lead to the development of clinical biomarkers that identify ARMS subgroups with increased risk of progressing into frank psychosis (McGuire et al. 2015).

\section{Limitations and future directions}

The present study had several limitations. First, although TBSS partially overcomes the misalignment of FA images in WM voxel-based analysis (Smith et al. 2006), WM fibers at the boundary of gray matter and WM, or deep in gray-matter regions, could be omitted from consideration. In addition, TBSS uses groupaveraged WM skeleton as the proxy of major WM tracts. Future works involving high-resolution diffusion imaging and WM tractography may help to examine the direct correspondence between FC and structural connectivity as well as minimize errors due to the inter-individual differences in WM structure. Moreover, the test-retest reliability of fMRI and DTI studies should be considered when interpreting our findings. While we have demonstrated ARMS-specific functional and structural dysconnectivity, good reproducibility is the prerequisite for successfully replicating these findings and transforming them into clinical biomarkers. A multisite test-retest study reported good reproducibility of diffusion metrics derived using TBSS (Jovicich et al. 2014). Similarly, (Song et al. 2012) used seed-based correlation method and found reliable and stable FC estimations in the group of young participants (mean ICC $=0.4$ ). However, intra- and interindividual test-retest variability do exist in task-free fMRI analysis and might be contributed to by endogenous neural dynamics and various non-neural factors such as head motion and scanner noise (Zuo \& Xing, 2014). In summary, based on a substance-free ARMS sample, we provide converging evidence, from both structural and functional connectivity analyses, to support the SN dysconnectivity hypothesis in individuals at risk for psychosis. The extent of SN disruption correlated with psychotic symptom severity and was more pronounced in subjects that developed psychosis, which can be utilized for prediction of psychosis transition.

\section{Supplementary material}

For supplementary material accompanying this paper visit http://dx.doi.org/10.1017/S0033291716001410.

\section{Acknowledgements}

The Singapore Translational and Clinical Research in Psychosis is supported by the National Research Foundation Singapore under the National Medical Research Council Translational and Clinical Research Flagship Program (grant no.: NMRC/TCR/003/2008). This research was supported by Biomedical Research Council (13/1/96/19/687), National Medical Research Council, Singapore (CIRG/1416/2015, CBRG/0088/ 2015), and Duke-NUS Medical School Signature Research Program funded by Ministry of Health, Singapore, by NIH grants (R01MH074794, P41EB015902, R01AG042512, R01MH102377) and by a NARSAD young investigator award.

\section{Declaration of Interest}

None.

\section{References}

Allen P, Luigjes J, Howes OD, Egerton A, Hirao K, Valli I, Kambeitz J, Fusar-Poli P, Broome M, McGuire P (2012). Transition to psychosis associated with prefrontal and subcortical dysfunction in ultra high-risk individuals. Schizophrenia Bulletin 38, 1268-1276.

Bloemen OJ, de Koning MB, Schmitz N, Nieman DH, Becker HE, de Haan L, Dingemans P, Linszen DH, van Amelsvoort TA (2010). White-matter markers for psychosis in a prospective ultra-high-risk cohort. Psychological Medicine 40, 1297-1304.

Boksman K, Theberge J, Williamson P, Drost DJ, Malla A, Densmore M, Takhar J, Pavlosky W, Menon RS, Neufeld RW (2005). A 4.0-T fMRI study of brain connectivity during word fluency in first-episode schizophrenia. Schizophrenia Research 75, 247-263.

Bracht T, Horn H, Strik W, Federspiel A, Razavi N, Stegmayer K, Wiest R, Dierks T, Muller TJ, Walther S (2014). White matter pathway organization of the reward system is related to positive and negative symptoms in schizophrenia. Schizophrenia Research 153, 136-142.

Buchsbaum MS, Friedman J, Buchsbaum BR, Chu KW, Hazlett EA, Newmark R, Schneiderman JS, Torosjan Y, Tang C, Hof PR, Stewart D, Davis KL, Gorman J (2006). Diffusion tensor imaging in schizophrenia. Biological Psychiatry 60, 1181-1187.

Carletti F, Woolley JB, Bhattacharyya S, Perez-Iglesias R, Fusar Poli P, Valmaggia L, Broome MR, Bramon E, Johns L, Giampietro V, Williams SC, Barker GJ, McGuire PK (2012). Alterations in white matter evident before the onset of psychosis. Schizophrenia Bulletin 38, 1170-1179.

Chudasama Y, Robbins TW (2006). Functions of frontostriatal systems in cognition: comparative neuropsychopharmacological studies in rats, monkeys and humans. Biological Psychology 73, 19-38.

Cortese S, Imperati D, Zhou J, Proal E, Klein RG, Mannuzza S, Ramos-Olazagasti MA, Milham MP, Kelly C, 
Castellanos FX (2013). White matter alterations at 33-year follow-up in adults with childhood attention-deficit/ hyperactivity disorder. Biological Psychiatry 74, 591-598.

Cox RW (1996). AFNI: software for analysis and visualization of functional magnetic resonance neuroimages. Computers and Biomedical Research 29, 162-173.

Craig AD (2009). How do you feel - now? The anterior insula and human awareness. Nature Reviews Neuroscience 10, 5970.

Dandash O, Fornito A, Lee J, Keefe RS, Chee MW, Adcock RA, Pantelis C, Wood SJ, Harrison BJ (2014). Altered striatal functional connectivity in subjects with an at-risk mental state for psychosis. Schizophrenia Bulletin 40, 904913.

Edward Roberts R, Curran HV, Friston KJ, Morgan CJ (2014). Abnormalities in white matter microstructure associated with chronic ketamine use. Neuropsychopharmacology 39, 329-338.

Federspiel A, Begre S, Kiefer C, Schroth G, Strik WK, Dierks T (2006). Alterations of white matter connectivity in first episode schizophrenia. Neurobiology of Disease 22, 702709.

Fornito A, Harrison BJ, Goodby E, Dean A, Ooi C, Nathan PJ, Lennox BR, Jones PB, Suckling J, Bullmore ET (2013). Functional dysconnectivity of corticostriatal circuitry as a risk phenotype for psychosis. JAMA Psychiatry 70, 11431151.

Fornito A, Yucel M, Patti J, Wood SJ, Pantelis C (2009). Mapping grey matter reductions in schizophrenia: an anatomical likelihood estimation analysis of voxel-based morphometry studies. Schizophrenia Research 108, 104-113.

Fornito A, Zalesky A, Pantelis C, Bullmore ET (2012). Schizophrenia, neuroimaging and connectomics. NeuroImage 62, 2296-2314.

Fox MD, Snyder AZ, Vincent JL, Corbetta M, Van Essen DC, Raichle ME (2005). The human brain is intrinsically organized into dynamic, anticorrelated functional networks. Proceedings of the National Academy of Sciences USA 102, 9673-9678.

Fusar-Poli P (2012). Voxel-wise meta-analysis of fMRI studies in patients at clinical high risk for psychosis. Journal of Psychiatry and Neuroscience 37, 106-112.

Fusar-Poli P, Bonoldi I, Yung AR, Borgwardt S, Kempton MJ, Valmaggia L, Barale F, Caverzasi E, McGuire P (2012). Predicting psychosis: meta-analysis of transition outcomes in individuals at high clinical risk. Archives of General Psychiatry 69, 220-229.

Fusar-Poli P, Howes OD, Allen P, Broome M, Valli I, Asselin MC, Grasby PM, McGuire PK (2010). Abnormal frontostriatal interactions in people with prodromal signs of psychosis: a multimodal imaging study. Archives of General Psychiatry 67, 683-691.

Greicius MD, Srivastava G, Reiss AL, Menon V (2004). Default-mode network activity distinguishes Alzheimer's disease from healthy aging: evidence from functional MRI. Proceedings of the National Academy of Sciences USA 101, 4637-4642.

Habets P, Marcelis M, Gronenschild E, Drukker M, van Os J, Genetic R, Outcome of P (2011). Reduced cortical thickness as an outcome of differential sensitivity to environmental risks in schizophrenia. Biological Psychiatry 69, 487-494.

Honey GD, Pomarol-Clotet E, Corlett PR, Honey RA, McKenna PJ, Bullmore ET, Fletcher PC (2005). Functional dysconnectivity in schizophrenia associated with attentional modulation of motor function. Brain 128, 25972611.

Hong Z, Ng KK, Sim SK, Ngeow MY, Zheng H, Lo JC, Chee MW, Zhou J (2015). Differential age-dependent associations of gray matter volume and white matter integrity with processing speed in healthy older adults. NeuroImage 123, $42-50$.

Hoptman MJ, Nierenberg J, Bertisch HC, Catalano D, Ardekani BA, Branch CA, Delisi LE (2008). A DTI study of white matter microstructure in individuals at high genetic risk for schizophrenia. Schizophrenia Research 106, 115-124.

Hua K, Zhang J, Wakana S, Jiang H, Li X, Reich DS, Calabresi PA, Pekar JJ, van Zijl PC, Mori S (2008). Tract probability maps in stereotaxic spaces: analyses of white matter anatomy and tract-specific quantification. NeuroImage 39, 336-347.

Jafri MJ, Pearlson GD, Stevens M, Calhoun VD (2008). A method for functional network connectivity among spatially independent resting-state components in schizophrenia. NeuroImage 39, 1666-1681.

Jenkinson M, Beckmann CF, Behrens TE, Woolrich MW, Smith SM (2012). Fsl. NeuroImage 62, 782-790.

Jiang L, Xu Y, Zhu XT, Yang Z, Li HJ, Zuo XN (2015). Local-to-remote cortical connectivity in early- and adulthoodonset schizophrenia. Translational Psychiatry 5, e566.

Jovicich J, Marizzoni M, Bosch B, Bartres-Faz D, Arnold J, Benninghoff J, Wiltfang J, Roccatagliata L, Picco A, Nobili F, Blin O, Bombois S, Lopes R, Bordet R, Chanoine V, Ranjeva JP, Didic M, Gros-Dagnac H, Payoux P, Zoccatelli G, Alessandrini F, Beltramello A, Bargallo N, Ferretti A, Caulo M, Aiello M, Ragucci M, Soricelli A, Salvadori N, Tarducci R, Floridi P, Tsolaki M, Constantinidis $\mathbf{M}$, Drevelegas A, Rossini PM, Marra C, Otto J, Reiss-Zimmermann M, Hoffmann KT, Galluzzi S, Frisoni GB (2014). Multisite longitudinal reliability of tract-based spatial statistics in diffusion tensor imaging of healthy elderly subjects. NeuroImage 101, 390-403.

Karlsgodt KH, Niendam TA, Bearden CE, Cannon TD (2009). White matter integrity and prediction of social and role functioning in subjects at ultra-high risk for psychosis. Biological Psychiatry 66, 562-569.

Kelly AM, Uddin LQ, Biswal BB, Castellanos FX, Milham MP (2008). Competition between functional brain networks mediates behavioral variability. Neurolmage 39, 527-537.

Klauser P, Zhou J, Lim JK, Poh JS, Zheng H, Tng HY, Krishnan R, Lee J, Keefe RS, Adcock RA, Wood SJ, Fornito A, Chee MW (2015). Lack of evidence for regional brain volume or cortical thickness abnormalities in youths at clinical high risk for psychosis: findings from the longitudinal youth at risk study. Schizophrenia Bulletin 41, 1285-1293.

Krolak-Salmon P, Henaff MA, Isnard J, Tallon-Baudry C, Guenot M, Vighetto A, Bertrand O, Mauguiere F (2003). 
An attention modulated response to disgust in human ventral anterior insula. Annals of Neurology 53, 446-453.

Kubicki M, McCarley R, Westin CF, Park HJ, Maier S, Kikinis R, Jolesz FA, Shenton ME (2007). A review of diffusion tensor imaging studies in schizophrenia. Journal of Psychiatric Research 41, 15-30.

Kunimatsu N, Aoki S, Kunimatsu A, Yoshida M, Abe O, Yamada H, Masutani Y, Kasai K, Yamasue H, Ohtsu H, Ohtomo K (2008). Tract-specific analysis of the superior occipitofrontal fasciculus in schizophrenia. Psychiatry Research 164, 198-205.

Kurth F, Zilles K, Fox PT, Laird AR, Eickhoff SB (2010). A link between the systems: functional differentiation and integration within the human insula revealed by meta-analysis. Brain Structure and Function 214, 519-534.

Laroi F, Luhrmann TM, Bell V, Christian Jr WA, Deshpande S, Fernyhough C, Jenkins J, Woods A (2014). Culture and hallucinations: overview and future directions. Schizophrenia Bulletin 40 (Suppl. 4), S213-S220.

Le Bihan D (2003). Looking into the functional architecture of the brain with diffusion MRI. Nature Reviews Neuroscience 4, 469-480.

Lee DY, Smith GN, Su W, Honer WG, Macewan GW, Lapointe JS, Vertinsky AT, Vila-Rodriguez F, Kopala LC, Lang DJ (2012). White matter tract abnormalities in first-episode psychosis. Schizophrenia Research 141, 29-34.

Leech R, Kamourieh S, Beckmann CF, Sharp DJ (2011). Fractionating the default mode network: distinct contributions of the ventral and dorsal posterior cingulate cortex to cognitive control. Journal of Neuroscience 31, 32173224.

Li HJ, Xu Y, Zhang KR, Hoptman MJ, Zuo XN (2015). Homotopic connectivity in drug-naive, first-episode, early-onset schizophrenia. Journal of Child Psychology and Psychiatry and Allied Disciplines 56, 432-443.

Li J, Kale Edmiston E, Chen K, Tang Y, Ouyang X, Jiang $Y$, Fan G, Ren L, Liu J, Zhou Y, Jiang W, Liu Z, Xu K, Wang F (2014). A comparative diffusion tensor imaging study of corpus callosum subregion integrity in bipolar disorder and schizophrenia. Psychiatry Research 221, 58-62.

Liang M, Zhou Y, Jiang T, Liu Z, Tian L, Liu H, Hao Y (2006). Widespread functional disconnectivity in schizophrenia with resting-state functional magnetic resonance imaging. Neuroreport 17, 209-213.

Liao Y, Tang J, Fornito A, Liu T, Chen X, Chen H, Xiang X, Wang X, Hao W (2012). Alterations in regional homogeneity of resting-state brain activity in ketamine addicts. Neuroscience Letters 522, 36-40.

Liu X, Lai Y, Wang X, Hao C, Chen L, Zhou Z, Yu X, Hong N (2013). Reduced white matter integrity and cognitive deficit in never-medicated chronic schizophrenia: a diffusion tensor study using TBSS. Behavioural Brain Research 252, 157-163.

Liu X, Lai Y, Wang X, Hao C, Chen L, Zhou Z, Yu X, Hong N (2014). A combined DTI and structural MRI study in medicated-naive chronic schizophrenia. Magnetic Resonance Imaging 32, 1-8.

Mamah D, Barch DM, Repovs G (2013). Resting state functional connectivity of five neural networks in bipolar disorder and schizophrenia. Journal of Affective Disorders 150, 601-609.

Mamah D, Conturo TE, Harms MP, Akbudak E, Wang L, McMichael AR, Gado MH, Barch DM, Csernansky JG (2010). Anterior thalamic radiation integrity in schizophrenia: a diffusion-tensor imaging study. Psychiatry Research 183, 144-150.

McGuire P, Sato JR, Mechelli A, Jackowski A, Bressan RA, Zugman A (2015). Can neuroimaging be used to predict the onset of psychosis? Lancet Psychiatry 2, 1117-1122.

Menon V, Uddin LQ (2010). Saliency, switching, attention and control: a network model of insula function. Brain Structure and Function 214, 655-667.

Mesulam MM, Mufson EJ (1982). Insula of the old world monkey. I. Architectonics in the insulo-orbito-temporal component of the paralimbic brain. Journal of Comparative Neurology 212, 1-22.

Minzenberg MJ, Laird AR, Thelen S, Carter CS, Glahn DC (2009). Meta-analysis of 41 functional neuroimaging studies of executive function in schizophrenia. Archives of General Psychiatry 66, 811-822.

Mutschler I, Wieckhorst B, Kowalevski S, Derix J, Wentlandt J, Schulze-Bonhage A, Ball T (2009). Functional organization of the human anterior insular cortex. Neuroscience Letters 457, 66-70.

Oertel-Knochel V, Linden DE (2011). Cerebral asymmetry in schizophrenia. Neuroscientist 17, 456-467.

Oh JS, Kubicki M, Rosenberger G, Bouix S, Levitt JJ, McCarley RW, Westin CF, Shenton ME (2009). Thalamo-frontal white matter alterations in chronic schizophrenia: a quantitative diffusion tractography study. Human Brain Mapping 30, 3812-3825.

Palaniyappan L, Liddle PF (2012). Does the salience network play a cardinal role in psychosis? An emerging hypothesis of insular dysfunction. Journal of Psychiatry and Neuroscience 37, 17-27.

Palaniyappan L, Simmonite M, White TP, Liddle EB, Liddle PF (2013). Neural primacy of the salience processing system in schizophrenia. Neuron 79, 814-828.

Pasternak O, Sochen N, Gur Y, Intrator N, Assaf Y (2009). Free water elimination and mapping from diffusion MRI. Magnetic Resonance in Medicine 62, 717-730.

Pasternak O, Westin CF, Bouix S, Seidman LJ, Goldstein JM, Woo TU, Petryshen TL, Mesholam-Gately RI, McCarley RW, Kikinis R, Shenton ME, Kubicki M (2012). Excessive extracellular volume reveals a neurodegenerative pattern in schizophrenia onset. Journal of Neuroscience 32, 17365-17372.

Perez-Iglesias R, Tordesillas-Gutierrez D, Barker GJ, McGuire PK, Roiz-Santianez R, Mata I, de Lucas EM, Quintana F, Vazquez-Barquero JL, Crespo-Facorro B (2010). White matter defects in first episode psychosis patients: a voxelwise analysis of diffusion tensor imaging. NeuroImage 49, 199-204.

Peters BD, Schmitz N, Dingemans PM, van Amelsvoort TA, Linszen DH, de Haan L, Majoie CB, den Heeten GJ (2009). Preliminary evidence for reduced frontal white matter integrity in subjects at ultra-high-risk for psychosis. Schizophrenia Research 111, 192-193. 
Pettersson-Yeo W, Allen P, Benetti S, McGuire P, Mechelli A (2011). Dysconnectivity in schizophrenia: where are we now? Neuroscience \& Biobehavioral Reviews 35, 1110-1124.

Power JD, Barnes KA, Snyder AZ, Schlaggar BL, Petersen SE (2012). Spurious but systematic correlations in functional connectivity MRI networks arise from subject motion. NeuroImage 59, 2142-2154.

Power JD, Barnes KA, Snyder AZ, Schlaggar BL, Petersen SE (2013). Steps toward optimizing motion artifact removal in functional connectivity MRI; a reply to Carp. NeuroImage 76, 439-441.

Price G, Bagary MS, Cercignani M, Altmann DR, Ron MA (2005). The corpus callosum in first episode schizophrenia: a diffusion tensor imaging study. Journal of Neurology, Neurosurgery and Psychiatry 76, 585-587.

Sabb FW, van Erp TG, Hardt ME, Dapretto M, Caplan R, Cannon TD, Bearden CE (2010). Language network dysfunction as a predictor of outcome in youth at clinical high risk for psychosis. Schizophrenia Research 116, 173-183.

Saze T, Hirao K, Namiki C, Fukuyama H, Hayashi T, Murai T (2007). Insular volume reduction in schizophrenia. European Archives of Psychiatry and Clinical Neuroscience 257, 473-479.

Schmidt A, Lenz C, Smieskova R, Harrisberger F, Walter A, Riecher-Rossler A, Simon A, Lang UE, McGuire P, Fusar-Poli P, Borgwardt SJ (2015). Brain diffusion changes in emerging psychosis and the impact of state-dependent psychopathology. Neuro-Signals 23, 71-83.

Schmidt A, Palaniyappan L, Smieskova R, Simon A, Riecher-Rossler A, Lang UE, Fusar-Poli P, McGuire P, Borgwardt SJ (2016). Dysfunctional insular connectivity during reward prediction in patients with first-episode psychosis. Journal of Psychiatry and Neuroscience 41, 150234.

Seeley WW, Menon V, Schatzberg AF, Keller J, Glover GH, Kenna H, Reiss AL, Greicius MD (2007). Dissociable intrinsic connectivity networks for salience processing and executive control. Journal of Neuroscience 27, 2349-2356.

Shim G, Oh JS, Jung WH, Jang JH, Choi CH, Kim E, Park HY, Choi JS, Jung MH, Kwon JS (2010). Altered resting-state connectivity in subjects at ultra-high risk for psychosis: an fMRI study. Behavioral and Brain Functions 6, 58.

Smith SM, Jenkinson M, Johansen-Berg $H$, Rueckert $D$, Nichols TE, Mackay CE, Watkins KE, Ciccarelli O, Cader MZ, Matthews PM, Behrens TE (2006). Tract-based spatial statistics: voxelwise analysis of multi-subject diffusion data. NeuroImage 31, 1487-1505.

Song J, Desphande AS, Meier TB, Tudorascu DL, Vergun S, Nair VA, Biswal BB, Meyerand ME, Birn RM, Bellec P, Prabhakaran V (2012). Age-related differences in test-retest reliability in resting-state brain functional connectivity. PLoS ONE 7, e49847.

Takahashi T, Wood SJ, Soulsby B, Tanino R, Wong MT, McGorry PD, Suzuki M, Velakoulis D, Pantelis C (2009). Diagnostic specificity of the insular cortex abnormalities in first-episode psychotic disorders. Progress in Neuro-Psychopharmacology and Biological Psychiatry 33, 651657.

Tang J, Liao Y, Zhou B, Tan C, Liu T, Hao W, Hu D, Chen X (2010). Abnormal anterior cingulum integrity in first episode, early-onset schizophrenia: a diffusion tensor imaging study. Brain Research 1343, 199-205.

Tognin S, Pettersson-Yeo W, Valli I, Hutton C, Woolley J, Allen P, McGuire P, Mechelli A (2013). Using structural neuroimaging to make quantitative predictions of symptom progression in individuals at ultra-high risk for psychosis. Front Psychiatry 4, 187.

Tu PC, Hsieh JC, Li CT, Bai YM, Su TP (2012).

Cortico-striatal disconnection within the cingulo-opercular network in schizophrenia revealed by intrinsic functional connectivity analysis: a resting fMRI study. NeuroImage 59, 238-247.

Uddin LQ (2015). Salience processing and insular cortical function and dysfunction. Nature Reviews Neuroscience 16, 55-61.

United Nations Office on Drugs and Crime (2008). World drug report 2008. United Nations Publication 1, 273-280.

Van der Werf YD, Jolles J, Witter MP, Uylings HB (2003). Contributions of thalamic nuclei to declarative memory functioning. Cortex 39, 1047-1062.

von Hohenberg CC, Pasternak O, Kubicki M, Ballinger T, Vu MA, Swisher T, Green K, Giwerc M, Dahlben B, Goldstein JM, Woo TU, Petryshen TL, Mesholam-Gately RI, Woodberry KA, Thermenos HW, Mulert C, McCarley RW, Seidman LJ, Shenton ME (2014). White matter microstructure in individuals at clinical high risk of psychosis: a whole-brain diffusion tensor imaging study. Schizophrenia Bulletin 40, 895-903.

Wager TD, Barrett LF (2004). From affect to control: functional specialization of the insula in motivation and regulation. Published online at PsycExtra (http://www.apa. org/pubs/databases/psycextra).

Wang F, Sun Z, Cui L, Du X, Wang X, Zhang H, Cong Z, Hong N, Zhang D (2004). Anterior cingulum abnormalities in male patients with schizophrenia determined through diffusion tensor imaging. American Journal of Psychiatry 161, 573-575.

Welch KA, McIntosh AM, Job DE, Whalley HC, Moorhead TW, Hall J, Owens DG, Lawrie SM, Johnstone EC (2011). The impact of substance use on brain structure in people at high risk of developing schizophrenia. Schizophrenia Bulletin 37, 1066-1076.

Williamson P (2007). Are anticorrelated networks in the brain relevant to schizophrenia? Schizophrenia Bulletin 33, 994-1003.

Wilmsmeier A, Ohrmann P, Suslow T, Siegmund A, Koelkebeck K, Rothermundt M, Kugel H, Arolt V, Bauer J, Pedersen A (2010). Neural correlates of set-shifting: decomposing executive functions in schizophrenia. Journal of Psychiatry and Neuroscience 35, 321-329.

Wood SJ, Reniers RL, Heinze K (2013). Neuroimaging findings in the at-risk mental state: a review of recent literature. Canadian Journal of Psychiatry/Revue Canadienne de Psychiatrie 58, 13-18.

Wotruba D, Michels L, Buechler R, Metzler S, Theodoridou A, Gerstenberg M, Walitza S, Kollias S, Rossler W, Heekeren K (2014). Aberrant coupling within and across the default mode, task-positive, and salience network in subjects at risk for psychosis. Schizophrenia Bulletin 40, 1095-1104. 
Yaakub SN, Dorairaj K, Poh JS, Asplund CL, Krishnan R, Lee J, Keefe RS, Adcock RA, Wood SJ, Chee MW (2013). Preserved working memory and altered brain activation in persons at risk for psychosis. American Journal of Psychiatry 170, 1297-1307.

Yang Z, Xu Y, Xu T, Hoy CW, Handwerker DA, Chen G, Northoff G, Zuo XN, Bandettini PA (2014). Brain network informed subject community detection in early-onset schizophrenia. Scientific Reports 4, 5549.

Yung AR, McGorry PD, McFarlane CA, Jackson HJ, Patton GC, Rakkar A (1996). Monitoring and care of young people at incipient risk of psychosis. Schizophrenia Bulletin 22, 283303.

Yung AR, Yuen HP, McGorry PD, Phillips LJ, Kelly D, Dell'Olio M, Francey SM, Cosgrave EM, Killackey E, Stanford C, Godfrey K, Buckby J (2005). Mapping the onset of psychosis: the comprehensive assessment of at-risk mental states. Australian and New Zealand Journal of Psychiatry 39, 964-971.
Zhou J, Greicius MD, Gennatas ED, Growdon ME, Jang JY, Rabinovici GD, Kramer JH, Weiner M, Miller BL, Seeley WW (2010). Divergent network connectivity changes in behavioural variant frontotemporal dementia and Alzheimer's disease. Brain 133, 1352-1367.

Zhou J, Seeley WW (2014). Network dysfunction in Alzheimer's disease and frontotemporal dementia: implications for psychiatry. Biological Psychiatry 75, 565-573.

Zhou Y, Liang M, Tian L, Wang K, Hao Y, Liu H, Liu Z, Jiang $T$ (2007). Functional disintegration in paranoid schizophrenia using resting-state fMRI. Schizophrenia Research 97, 194-205.

Zuo XN, Ehmke R, Mennes M, Imperati D, Castellanos FX, Sporns O, Milham MP (2012). Network centrality in the human functional connectome. Cerebral Cortex 22, 1862-1875.

Zuo XN, Xing XX (2014). Test-retest reliabilities of resting-state FMRI measurements in human brain functional connectomics: a systems neuroscience perspective. Neuroscience \& Biobehavioral Reviews 45, 100-118. 